\title{
Single User MAC Level Throughput Comparision: IEEE 802.11ax vs. IEEE 802.11ac
}

\author{
Oran Sharon1, Yaron Alpert² \\ ${ }^{1}$ Department of Computer Science, Netanya Academic College, 1 University Street, Netanya, Israel \\ ${ }^{2}$ Intel, Ra'anana, Israel \\ Email:oran@math.haifa.ac.il, Yaron.Alpert@intel.com
}

How to cite this paper: Sharon, O. and Alpert, Y. (2017) Single User MAC Level Throughput Comparision: IEEE 802.11ax vs. IEEE 802.11ac. Wireless Sensor Network, 9, 166-177.

https://doi.org/10.4236/wsn.2017.95009

Received: March 27, 2017

Accepted: May 24, 2017

Published: May 27, 2017

Copyright ( 92017 by authors and Scientific Research Publishing Inc. This work is licensed under the Creative Commons Attribution International License (CC BY 4.0).

http://creativecommons.org/licenses/by/4.0/

(c) (i) Open Access

\begin{abstract}
With the ever-increasing range of video and audio applications in portable handheld devices, demand for high throughput in Wi-Fi networks is escalating. In this paper we introduce several novel features defined in next generation WLAN, termed as IEEE 802.11ax standard, and compare between the maximum throughputs received in IEEE 802.11ax and IEEE 802.11ac in a scenario where the AP continuously transmits to one station in the Single User mode. The comparison is done as a function of the modulation/coding schemes in use. In IEEE 802.11ax we consider two levels of frame aggregation. IEEE 802.11 ax outperforms IEEE 802.11 ac by about $29 \%$ and $48 \%$ in reliable and unreliable channels respectively.
\end{abstract}

\section{Keywords}

IEEE 802.11ax, IEEE 802.11ac, Throughput, Single User, Aggregation

\section{Introduction}

The latest IEEE 802.11 Standard (Wi-Fi), created and maintained by the IEEE LAN/MAN Standards Committee (IEEE 802.11) [1] is currently the most effective solution within the range of Wireless Local Area Networks (WLAN). Since its first release in 1997 the standard provides the basis for Wireless network products using the Wi-Fi brand, and has since been improved upon in many ways. One of the main goals of these improvements is to increase the throughput achieved by users and to improve the Quality-of-Service (QoS) capabilities of the network.

To fulfill the promise of increasing IEEE 802.11 performance and QoS capabilities, a new amendment, IEEE 802.11ax, also known as High Efficiency (HE) WLAN, was introduced recently [2]. IEEE 802.11ax is a sixth generation of WLAN in the IEEE 802.11 set of WLANs [1] and it is a successor to IEEE 
802.11ac [3] [4]. IEEE 802.11ax is predicted to have maximum capacity of around $9.5 \mathrm{Gbps}$ in 2.4 and/or $5 \mathrm{GHz}$ and has the goal of providing 4 times the throughput of IEEE 802.11ac [5] [6] [7] [8].

The performance of IEEE 802.11ax has been investigated in only few papers up to now. For example, in [9] the authors assume a network with legacy and IEEE 802.11ax stations and examine fairness issues between the two sets of stations. In [10] the authors suggest an access protocol over the Uplink of an IEEE 802.11ax WLAN based on Multi User Multiple-Input-Multiple-Output (MU-MIMO) and OFDMA PHY.

In this paper we compare between the throughputs of IEEE 802.11ax and IEEE 802.11ac in a scenario where the AP continuously transmits UDP like traffic to a single station using the Single User (SU) operation mode. The AP transmits without collisions using advanced modulation and coding (MCS) schemes and using frame aggregation. This is one of the aspects to compare between new amendments of the IEEE 802.11 standard [11].

The paper is organized as follows: In Section 2 we describe the new features of IEEE 802.11ax relevant to this paper. In Section 3 we describe the transmission scenario over which we compare between IEEE 802.11ax and IEEE 802.11ac in the SU mode. In this mode we assume that the AP transmits continuously to a single station. In Section 4 we analytically compute the throughput of the SU mode and in Section 5 we present the throughputs of various protocols for the SU mode and compare between them. In Section 6 we analytically compute the PHY rates from which using a 256 MAC Protocol Data Units (MPDU) window size in IEEE 802.11ax is better than using a 64 MPDUs acknowledgment window size. Section 7 summarizes the paper. In the Appendix we derive the optimal number of MPDUs for the aggregation used in Section 6. In the rest of the paper we denote IEEE 802.11ac and IEEE 802.11ax by 11 ac and 11ax respectively.

\section{Frame Aggregation in IEEE 802.11ax}

In order to achieve the 4 times throughput compared to IEEE 802.11ac, the IEEE 802.11ax addresses several new features. We introduce some of these features below. We assume that the reader is familiar with the basics of the PHY and MAC layers of IEEE 802.11 described in previous papers, e.g. [12].

Assuming an OFDM based PHY layer, every OFDM symbol duration is extended from $3.2 \mu \mathrm{s}$ in 11 ac to $12.8 \mu \mathrm{s}$ in $11 \mathrm{ax}$. Since the same Guard Interval (GI) is added to every such symbol, the overhead in 11ax due to the GI is smaller than in 11ac. Second, in 11ax there are two new MCSs, 1024 QAM 3/4 and 1024 QAM 5/6, denoted MCS10 and MCS11 respectively, applicable for channels with bandwidth larger than $20 \mathrm{MHz}$. The above two features enhance the PHY rate of $11 \mathrm{ax}$.

In this paper we focus on the two-level frame aggregation scheme, in which several MPDUs are transmitted within a single PHY Service Data Unit (PSDU). Such a PSDU is denoted an Aggregate MAC Protocol Data Unit (A-MPDU) frame. In two-level aggregation every MPDU contains several MAC Service Data 
Units (MSDU). MPDUs are separated by an MPDU Delimiter field of 4 bytes and each MPDU contains MAC Header and Frame Control Sequence (FCS) fields. MSDUs within an MPDU are separated by a sub-header field of 14 bytes. Every MSDU is rounded to an integral multiple of 4 bytes together with the sub-header field. Every MPDU is also rounded to an integral multiple of 4 bytes. In $11 \mathrm{ax}$ and $11 \mathrm{ac}$, the size of an MPDU is limited to 11,454 bytes. In 11ac, an A-MPDU is limited to $1,048,575$ bytes and in $11 \mathrm{ax}$ it is limited to $4,194,304$ bytes. In both 11ac and 11ax, the transmission time of the PPDU (PSDU and its preamble) is limited to $\sim 5.4 \mathrm{~ms}(5400 \mu \mathrm{s})$ due to L-SIG (one of the legacy preamble's fields) duration limit [4].

In this paper we also assume that all the MPDUs transmitted in an A-MPDU frame are from the same Traffic Stream (TS). In this case up to 256 MPDUs are allowed in an A-MPDU frame of 11ax, while in 11ac up to only 64 MPDUs are allowed.

\section{Single User Model}

In the SU operation mode every transmitted PPDU is destined to one user only. We assume the traffic pattern shown in Figure 1 where the AP continuously transmits Data MSDUs to a station, and the station responds with the Block Ack (BAck) control frame [1]. A transmission of a PPDU from the AP followed by a BAck control frame from the station is denoted Transmission Cycle and such a cycle repeats itself continuously, as shown in Figure 1 . In one case the AP transmits up to 64 MPDUs in every A-MPDU frame and the BAck frame is 30 bytes long. The second case is relevant to 11ax only where the AP can transmit up to 256 MPDUs in an A-MPDU frame and so the BAck frame is 54 bytes long. The BAck frame is transmitted in the UL using the legacy mode (i.e. the mode used in the first generation of IEEE 802.11 WLANs) by using the legacy PHY basic rates' set. The UL PHY rate is set to the largest possible PHY rate in the set that is smaller or equal to the DL data rate.

In Figure 2 we show the PPDU formats used. In Figure 2(a) and Figure 2(b) we show the PPDU formats used over the DL by 11 ac and 11ax respectively. In the PPDU format of 11ac there are the VHT-LTF fields, the number of which equals to the number of Spatial Streams (SS) in use and the duration of each field is $4 \mu$ s. In the 11ax PPDU format, there are the HE-LTF fields, the number of which equals to the number of SSs in use. In this paper we assume that each

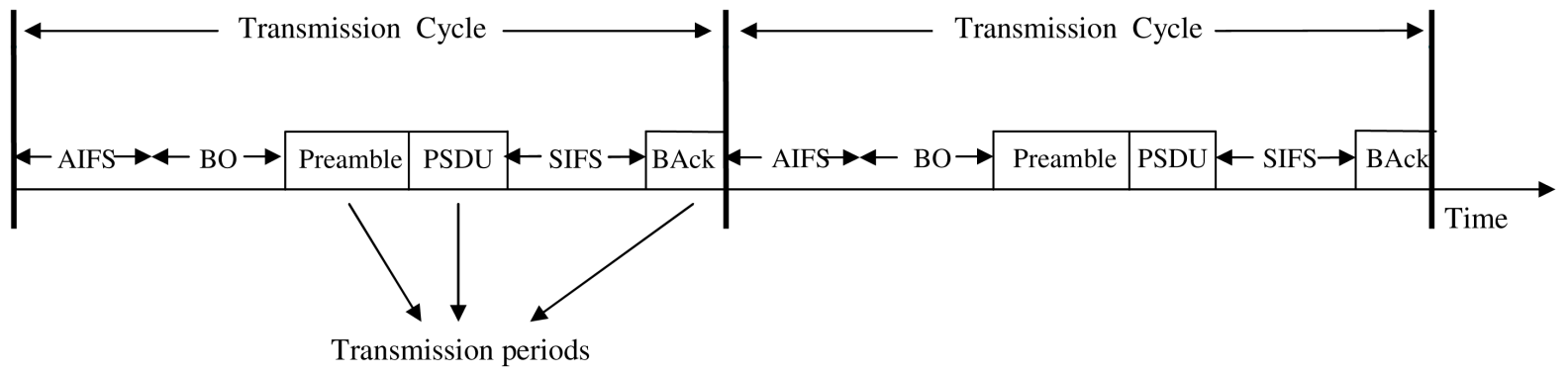

Figure 1. Transmissions from the AP to stations in single user mode in IEEE 802.11ac and in IEEE 802.11ax. 


\begin{tabular}{|c|c|c|c|c|c|c|c|c|c|}
\hline \multirow[b]{2}{*}{$8 \mu \mathrm{s}$} & \multirow[b]{2}{*}{$8 \mu \mathrm{s}$} & \multirow[b]{2}{*}{$4 \mu \mathrm{s}$} & \multirow[b]{2}{*}{$8 \mu \mathrm{s}$} & \multirow[b]{2}{*}{$4 \mu \mathrm{s}$} & \multicolumn{3}{|c|}{ Variable durations per VHT-LTF svmbol } & & \\
\hline & & & & & $4 \mu \mathrm{s}$ & & $4 \mu \mathrm{s}$ & & \\
\hline L-STF & L-LTF & L-SIG & VHT-SIG-A & VHT-STF & VHT-LTF & $\cdots$. & VHT-LTF & VHT-SIG-B & Data \\
\hline
\end{tabular}

(a) VHT (IEEE 802.11ac) AP DL SU PPDU format

Variable durations per HE-LTF svmbol

\begin{tabular}{c|c|c|c|c|c|c|c|c|c|c|}
\multicolumn{1}{c}{$8 \mu \mathrm{s}$} & $8 \mu \mathrm{s}$ & $4 \mu \mathrm{s}$ & $4 \mu \mathrm{s}$ & $8 \mu \mathrm{s}$ & $4 \mu \mathrm{s}$ & $7.2 \mu \mathrm{s}$ & \multicolumn{3}{c|}{$7.2 \mu \mathrm{s}$} \\
\hline L-STF & L-LTF & L-SIG & RL-SIG & HE-SIG-A & HE-STF & HE-LTF & $\cdots$ & HE-LTF & Data & PE \\
\hline
\end{tabular}

(b) HE (IEEE 802.11ax) AP DL SU PPDU format

Figure 2. The PPDU format in single user (SU) mode in IEEE 802.11ac and IEEE 802.11ax.

such field is composed of $2 \mathrm{X} \mathrm{LTF}$ and therefore it is of duration $7.2 \mu$ s [2]. Notice that in SU mode and when using the same number $S$ of SS, the preamble in $11 \mathrm{ax}$ is longer than that in 11 ac by $4 \mu \mathrm{s}+S \cdot(7.2-4) \mu \mathrm{s}=4 \mu \mathrm{s}+S \cdot 3.2 \mu \mathrm{s}$.

In Figure 2(b) we also show the legacy preamble format used by both $11 \mathrm{ac}$ and 11ax over the UL.

We assume the best effort Access Category in which $A I F S=43 \mu \mathrm{s}$, SIFS $=16 \mu \mathrm{s}$ and $C W_{\min }=16$ for an AP transmission. For an initial transmission the BackOff $(\mathrm{BO})$ interval is a random number being chosen uniformly from the range $\left[0, \cdots, C W_{\min }-1\right]$. Since we consider a very "large" number of transmissions from the AP we take the $\mathrm{BO}$ average value of $\left\lceil\frac{C W_{\min }-1}{2}\right\rceil$ and the average $\mathrm{BO}$ interval is $\left\lceil\frac{C W_{\min }-1}{2}\right\rceil$. SlotTime which equals $67.5 \mu$ s for a SlotTime $=9 \mu \mathrm{s}$. We also assume that the MAC Header is of 28 bytes and the FCS is of 4 bytes.

In the $5 \mathrm{GHz}$ band we assume a $160 \mathrm{MHz}$ channel $\mathrm{BW}$ and the AP has 4 antennas and every station has 1 antenna. Therefore, we assume 4 Spatial Streams (SS) and in this case, the PHY rates in the various MCSs and the preambles can be found in [2].

Finally, we consider several channel conditions that are expressed by different values of the Bit Error Rate (BER). We assume a model where these probabilities are bit-wise independent [13].

\section{Throughput Analysis: Single User Mode}

Let $X$ be the number of MPDU frames in an A-MPDU frame, numbered $1, \cdots, X$, and $Y_{i}$ be the number of MSDUs in MPDU number $i$. Let $L_{\text {DATA }}$ be the length, in bytes, of the MSDU frames.

Let MPDUDelimiter, MacHeader and FCS be the lenghs, in bytes, of the MAC Delimiter, MAC Header and FCS fields respectively. Also, let

$O_{M}=$ MPDUDelimiter + MacHeader + FCS, Len $=4 \cdot\left\lceil\frac{L_{D A T A}+14}{4}\right\rceil$ and $C_{i}=8 \times 4 \cdot\left\lceil\frac{O_{M}+Y_{i} \cdot \text { Len }}{4}\right\rceil$.

Then, the throughput in both $11 \mathrm{ax}$ and 11ac is given by Equation (1) [12] 
where BER is the Bit Error Rate, and $C_{i}$ defined above is the length of MPDU number $i$ in bits:

$$
T h r=\frac{\sum_{i=1}^{X} 8 \cdot Y_{i} \cdot L_{D A T A} \cdot(1-B E R)^{C_{i}}}{A I F S+B O+P_{D L}+T(D A T A)+S I F S+P_{U L}+T(B A c k)}
$$

where:

$$
\begin{aligned}
& T(\text { DATA })=T \text { Sym } \cdot\left\lceil\frac{\sum_{i=1}^{X} C_{i}+22}{T S y m \cdot R_{D L}}\right\rceil \\
& T(\text { BAck })=T \text { Sym } \cdot\left\lceil\frac{(30 \times 8)+22}{T S y m \cdot R_{U L}}\right\rceil
\end{aligned}
$$

$T(D A T A)$ and $T(B A c k)$ are the transmission times of the Data A-MPDU frames and the BAck frames respectively. Considering $T$ (BAck) it is based on the BAck frame's lengths. When assuming 30 bytes we consider the possibility of acknowledging 64 MPDUs in the BAck. In 11ax it is also possible to acknowledge 256 MPDUs and in this case the 30 bytes in $T$ (BAck) are replaced by 54 bytes.

TSym is the length of an OFDM symbol and every transmission must be of an integral number of OFDM symbols. The additional 22 bits in T(DATA) and $T(B A c k)$ in the denomination are due to the SERVICE and TAIL fields that are added to every transmission by the PHY layer convolutional protocol [4]. $R_{D L}$ and $R_{U L}$ are the DL and UL PHY rates respectively and $P_{D L}$ and $P_{U L}$ are the preambles used in the DL and UL respectively, see Figure 2.

The term in Equation (1) is not continuous and so it is difficult to find the optimal $X$ and $Y_{i}(\mathrm{~s})$, i.e. the values for $X$ and $Y_{i}(\mathrm{~s})$ that maximize the throughput. However, in [12] it is shown that if one neglects the rounding in the denomination of Equation (1) then the optimal solution has the property that all the MPDUs contain almost the same number of MSDUs: the difference between the largest and smallest number of MSDUs in MPDUs is at most 1 . The difference is indeed 1 if the limit on the transmission time of the PPDU does not enable to transmit the same number of MSDUs in all the MPDUs.

If one neglects the rounding of the denomination of Equation (1) the received throughput for every $X$ and $Y$, the number of MSDUs in every MPDU, is as large as that received in Equation (1). The difference depends on the size of the denomination.

We therefore use the result in [12] and look for the maximum throughput as follows: We check for every $X, 1 \leq X \leq 64$ (also $1 \leq X \leq 256$ for 11ax) and for every $Y, 1 \leq Y \leq Y_{\max }$, the received throughput such that $Y_{\max }$ is the maximum possible number of MSDUs in an MPDU. This is computed taking into account the upper limit of $5.4 \mathrm{~ms}$ on the transmission time of the PPDU (PSDU + preamble). In case where it is not possible to transmit the same number of MSDUs in all the MPDUs, part of the MPDUs have one more MSDU than the others, up to the above upper limit on the transmission time. We found that the smallest denomination of any of the maximum throughputs is around 1000 $\mu s$. Neglecting the rounding in the denomination reduces its size by at most 
$2 \times 13.6 \mu \mathrm{s}$ in $11 \mathrm{ax}$ and $2 \times 4 \mu \mathrm{s}$ in $11 \mathrm{ac}$. Thus, the error in the received maximum throughputs is in the order of at most $2.8 \%$.

\section{Throughput Results: Single User Mode}

In Figure 3 and Figure 4 we show the maximum throughput of 11ax and 11ac for two different channel conditions, $B E R=0$ and $B E R=10^{-5}$ respectively. Each figure contains results for 3 different sizes $L_{D A T A}$ of MSDUs: $L_{D A T A}=64$, 512 and 1500 bytes in parts (a), (b) and (c) respectively. There are results for $11 \mathrm{ac}$ with 64 MPDUs in every A-MPDU frame, for $11 \mathrm{ax}$ with 64 MPDUs in every A-MPDU frame and for 11ax with 256 MPDUs in every A-MPDU frame. The last two flavors of $11 \mathrm{ax}$ are denoted $11 \mathrm{ax} / 64$ and 11ax/256 respectively.

First, notice that in every figure the throughput is shown as a function of the MCSs in the $\mathrm{x}$-axis. In every MCS $11 \mathrm{ac}$ and $11 \mathrm{ax}$ enable different $\mathrm{PHY}$ rates and so the comparison is done as a function of the MCSs in use. Also notice that

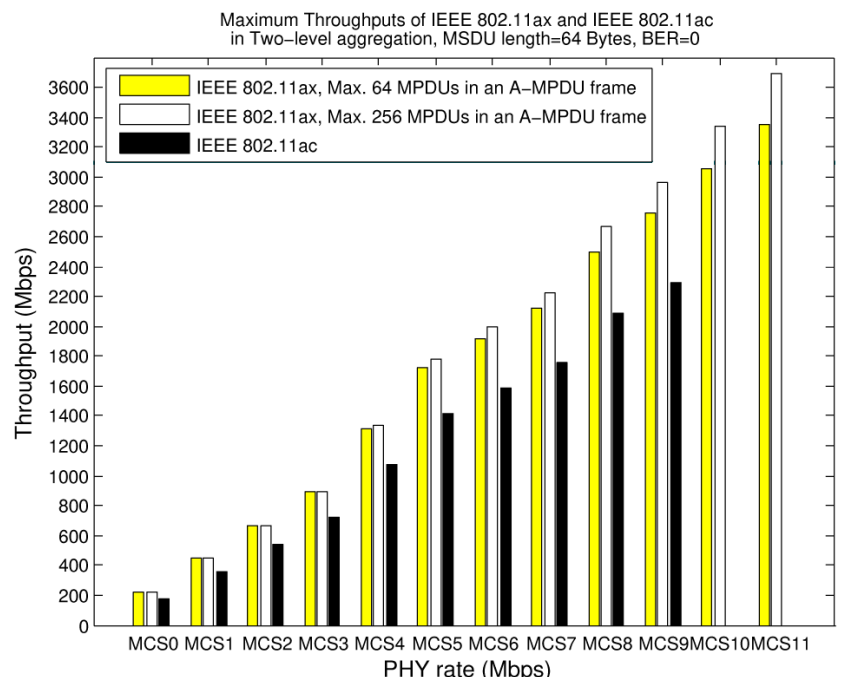

(a)

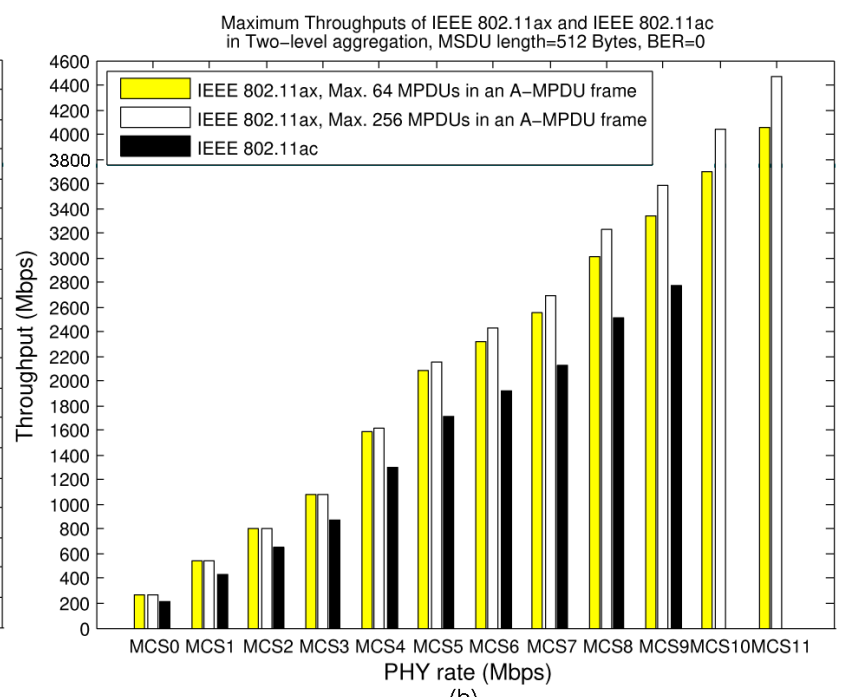

(b)

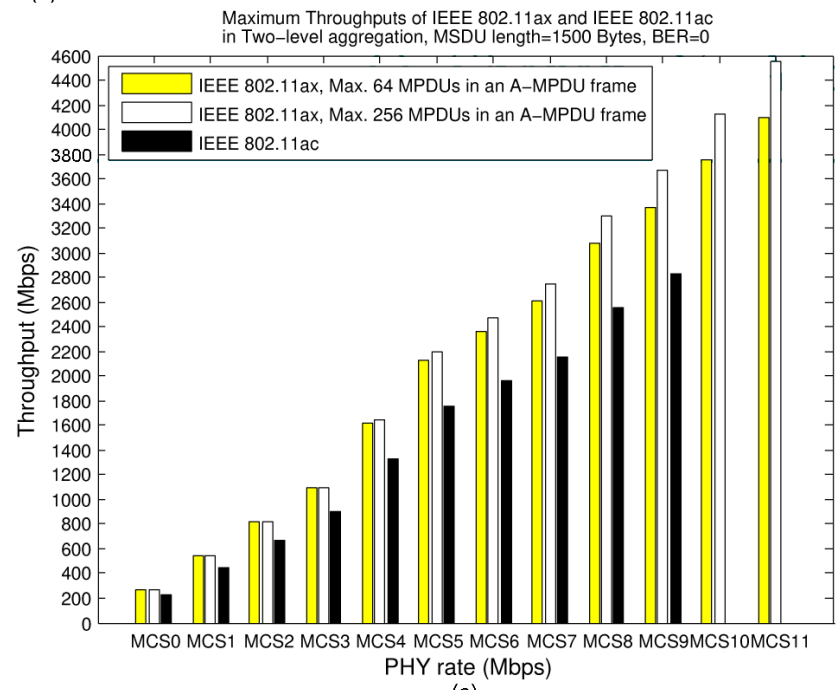

(c)

Figure 3. Comparison between the maximum throughputs of IEEE 802.11ax and IEEE 802.11ac in the two-level aggregation scheme, single user operation mode. $B E R=0$. 


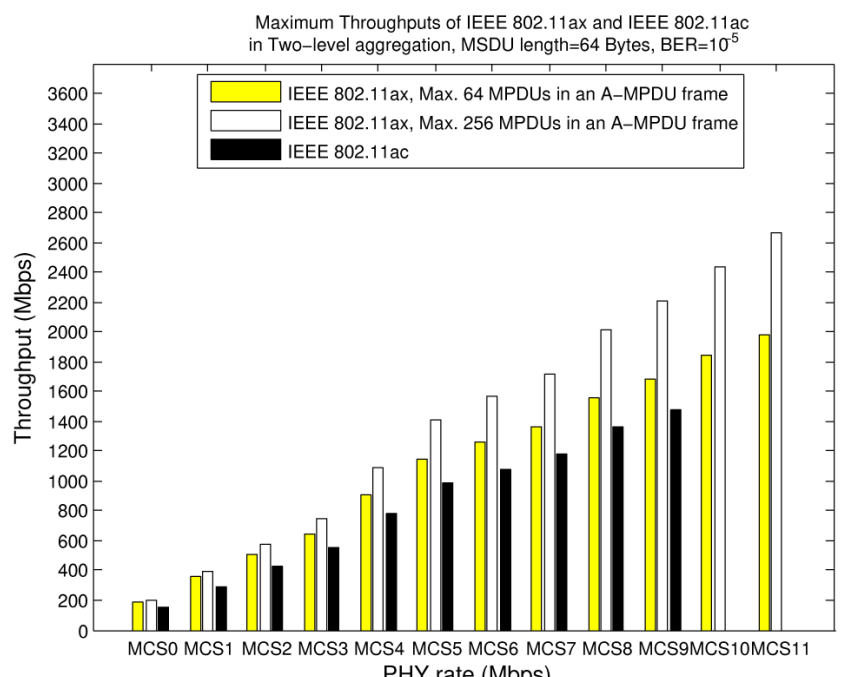

PHY rate (Mbps)

(a)

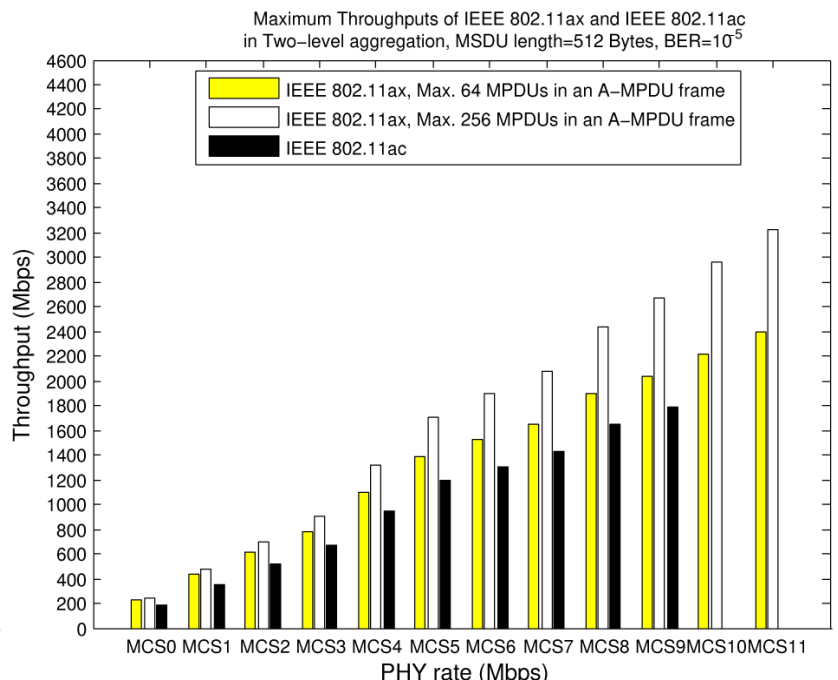

(b)

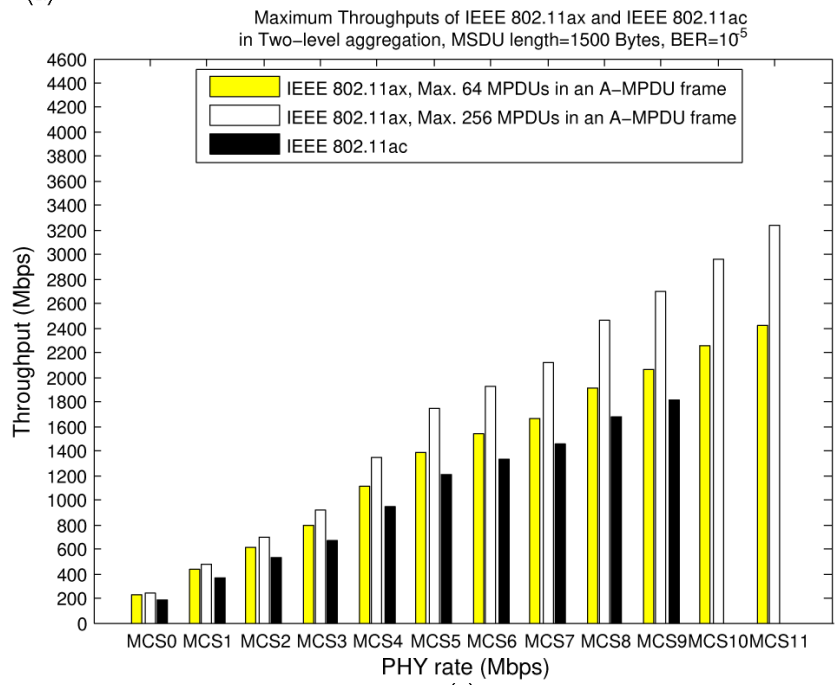

(c)

Figure 4. Comparison between the maximum throughputs of IEEE 802.11ax and IEEE 802.11ac in the two-level aggregation scheme, single user operation mode. $B E R=10^{-5}$.

MCS10 and MCS11 are not possible in 11ac and so 11ac does not have results for these MCSs.

In all the figures the performance of $11 \mathrm{ax}$ is better than that of $11 \mathrm{ac}$. This is due to the larger PHY rates that 11ax enables in every MCS compared to 11ac. For $B E R=0,11 \mathrm{ax} / 256$ outperforms $11 \mathrm{ac}$ by $29 \%$ and in $B E R=10^{-5}$ the improvement reaches $48 \%$. When comparing between $11 \mathrm{ax} / 64$ and 11ax/256 one can see that for $B E R=0 \quad 11 \mathrm{ax} / 256$ outperforms $11 \mathrm{ax} / 64$ only for MCSs larger than MCS2. On the other hand, in the case of $B E R=10^{-5} \quad 11 \mathrm{ax} / 256$ outperforms $11 \mathrm{ax} / 64$ starting from MCS0. The reason for this difference is as follows: for $B E R=0$ it is worth to transmit MPDUs with as much MSDUs as possible. Thus, not many MPDUs are transmitted when the maximum throughput is received and the limiting parameter on the throughput is the limit on the PPDU transmission time. Therefore, for small PHY rates, i.e. small MCSs, 11ax/256 has no advantage over 11ax/64. Only when the PHY rates increase the limit of 64 
MPDUs in 11ax/64 begins to be significant and 11ax/256 begins to outperform $11 \mathrm{ax} / 64$. When $B E R=10^{-5}$ it is worth to transmit short MPDUs because the failure probability of an MPDU increases with its length. In small PHY rates, the limiting parameter is now the number of MPDUs and not the limit on the PPDU transmission times. Therefore, 11ax/256 outperforms 11ax/64 also in small indexed MCSs.

Notice that $11 \mathrm{ax} / 256$ outperforms $11 \mathrm{ac}$ in $B E R=10^{-5}$, in percentage, more than in $B E R=0$. In $B E R=10^{-5}$ and $11 \mathrm{ax} / 256$ the MPDUs are much shorter than in $11 \mathrm{ac}$, i.e. contain a smaller number of MSDUs, in order to maintain on larger transmission success probabilities. More MPDUs in $B E R=10^{-5}$ enables the transmission of more MSDUs and are therefore significant. In $B E R=0$ MPDUs are large with relatively many MSDUs in both $11 \mathrm{ax} / 256$ and $11 \mathrm{ac}$ and given the same overhead (AIFS $\left.+B O+P_{D L}+S I F S+P_{U L}+T(B A c k)\right)$ most of the throughput is received when using not "too" many MPDUs. The larger number of MPDUs that $11 \mathrm{ax} / 256$ enables is therefore less significant than in $B E R=10^{-5}$ and so is the relative improvement in throughput between 11ax/256 and $11 \mathrm{ac}$.

A summarizing conclusion from the above is that 11ax outperforms $11 \mathrm{ac}$ in an unreliable channel more than in a reliable channel because 11 ax enables more MPDUs in a transmission. These MPDUs can be short in order to maintain a large success probability; thus, 11ax enables many short MPDUs, with a relatively large success probability, a feature that is not possible in $11 \mathrm{ac}$.

\section{Acknowledgment Window Size Analysis-Single User Mode}

One can conclude the following from the results in Section 5: First, as the BER is larger, 11ax/256 outperforms 11ax/64 from smaller PHY rates. Second, the MCS from which 11ax/256 outperforms 11ax/64 is not dependent on the MSDU size. We want to investigate these phenomena further.

In the following analysis, we use the above mentioned approximation from [12] where we neglect the rounding in the denomination of Equation (1) and assume that all the MPDUs contain the same number of MSDUs. We also neglect the rounding of the MPDU size and the addition of the 22 bits in the denomination for Data/Ack transmissions. Following this approximation Equation (1) turns out to be Equation (3):

$$
T h r=\frac{8 \cdot X \cdot Y \cdot L_{D A T A} \cdot(1-B E R)^{8 \cdot\left(O_{M}+Y \cdot \text { Len }\right)}}{O_{P}+\frac{8 \cdot X \cdot\left(O_{M}+Y \cdot \text { Len }\right)}{R_{D L}}}
$$

where $O_{P}=A I F S+B O+P_{D L}+S I F S+P_{U L}+\frac{(30 \times 8)+22}{R_{U L}}$.

Notice from Equation (3) that given a number $Y$ of MSDUs in an MPDU, it is worthwhile to contain as many MPDUs as possible in the A-MPDU frame, up to the limit on the PPDU transmission time. 


\subsection{Reliable Channel, $B E R=0$}

Let $\mathrm{MCS}_{\mathrm{C}}$ be the MCS from which 11ax/256 outperforms 11ax/64. Recall that $O_{M}$ is the sum of the lengths of the MPDU Delimiter, MAC Header and the FCS fields in bytes. Also recall that Len $=4 \cdot\left\lceil\frac{L_{D A T A}+14}{4}\right\rceil, R_{D L}$ be the DL PHY rate and let $T$ be the limit on the transmission time of the PPDU (5400 $\mu$ s in our case). Finally, let $Y_{\max }=\left\lfloor\frac{11454-O_{M}}{\text { Len }}\right\rfloor$ be the maximum possible number of MSDUs per MPDU frame. For $B E R=0$ it is most efficient to include $Y_{\max }$ MSDUs per MPDU frame and as many MPDUs in the A-MPDU frame up to the limit $T$. Then, one receives the following equation for 11ax/64 assuming that the PHY rate enables to transmit 64 MPDUs of $Y_{\max }$ MSDUs each: $T=\frac{8 \times 64 \cdot\left(O_{M}+Y_{\max } \cdot \text { Len }\right)}{R_{D L}}+O_{P}$. The largest DL PHY rate that enables the transmissions of up to 64 MPDUs is $R_{D L}=\frac{8 \times 64 \cdot\left(O_{M}+Y_{\max } \cdot \text { Len }\right)}{T-O_{P}}$.

Neglecting the rounding of $Y_{\max }$ we take $O_{M}+Y_{\max } \cdot$ Len $=11454$ bytes and so $R_{D L}=\frac{8 \times 64 \times 11545}{T-O_{P}}$ which, independently of $L_{D A T A}$, equals $1137 \mathrm{Mbps}$ for $T=5400 \mu \mathrm{s}$ and for the values of the other parameters in $O_{p}$ that we use in this paper. This DL PHY rate falls between MCS2 and MCS3 i.e., 11ax/256 outperforms 11ax/64 starting from $\mathrm{MCS}_{\mathrm{C}}=\mathrm{MCS} 3$ for any MSDU length $L_{D A T A}$. In Figure 3 the difference between $11 \mathrm{ax} / 64$ to $11 \mathrm{ax} / 256$ in MCS3 is too small to be noticed, however from MCS4 the difference is noticeable.

\subsection{Unreliable Channel, $B E R>0$}

For positive BERs the optimal number of MSDUs per MPDU is not necessarily $Y_{\max }$. Therefore, we use the following approximation. Given that it is worthwhile to transmit as long PPDUs as possible, then let $X_{\text {opt }}$ and $Y_{\text {opt }}$ be the number of MPDUs and the number of MSDUs per MPDU respectively in the optimal $\mathrm{A}-\mathrm{MPDU}$, i.e. the A-MPDU that achieves the largest throughput. Then, Equations (4) and (5) can give a relation between $X_{\text {opt }}$ and $Y_{\text {opt }}$ :

$$
T=\frac{X_{o p t} \cdot\left(Y_{o p t} \cdot \text { Len }+O_{M}\right)}{R_{D L}}+O_{P}
$$

Alternatively:

$$
Y_{\text {opt }}=\frac{R_{D L} \cdot T-R_{D L} \cdot O_{P}-X_{o p t} \cdot O_{M}}{X_{o p t} \cdot \text { Len }}
$$

Using Equations (4) and (5) the search for the optimal A-MPDU can consider only the number $X$ of MPDUs and the number $Y$ of MSDUs per MPDU that maintain Equation (5). We can therefore re-write Equation (3) as:

$$
T h r=\frac{8 \cdot X \cdot\left(\frac{R_{D L} \cdot T-R_{D L} \cdot O_{P}-X \cdot O_{M}}{X \cdot L e n}\right) \cdot L_{D A T A} \cdot(1-B E R)^{8 \cdot\left(O_{M}+\left(\frac{R_{D L} \cdot T-R_{D L} \cdot O_{P}-X \cdot O_{M}}{X \cdot \operatorname{Len}}\right) \cdot \operatorname{Len}\right)}}{T+O_{P}-P_{D L}}(6)
$$


Notice that the denomination of Equation (6) is a constant because we use the outcome that it is most efficient that the transmission time of the PPDU will be the largest possible.

To find the largest throughput we derive Equation (6) according to $X$ and find that the optimal $X$ is the single positive solution of a quadratic equation, which reveals that Equation (6) is unimodal. The optimal $X, X_{o p t}$, is given by Equation (7); we derive $X_{\text {opt }}$ in the Appendix.

$$
\begin{aligned}
X_{\text {opt }}= & \frac{L_{D A T A}}{\text { Len }} \cdot \frac{8}{\left(T+O_{P}-P_{D L}\right)} \cdot \frac{8 \cdot R_{D L} \cdot\left(T-O_{P}\right) \cdot \ln (1-B E R)}{2} \\
& \cdot\left(1-\sqrt{1-\frac{4}{8 \cdot O_{M} \cdot \ln (1-B E R)}}\right)
\end{aligned}
$$

If we now substitute the parameters in Equation (7) by the values we use in this paper, and using $B E R=10^{-5}$, we get that $X_{\text {opt }}=0.9678 \cdot R_{D L} \cdot X_{o p t}$ does not depend on the MSDU size but it is a function of the PHY rate $R_{D L}$. If we look for the PHY rates for which $X_{\text {opt }}>64$, i.e. $11 \mathrm{ax} / 256$ outperforms $11 \mathrm{ax} / 64$, we get the PHY rate of $66 \mathrm{Mbps}$. This means that the corresponding $\mathrm{MCS}_{\mathrm{C}}$ is MCS0 as is shown in Figure 4. Notice that by the above in turns out that the $\mathrm{MCS}_{\mathrm{C}}$ does not depend on the MSDUs' sizes, as it is also observed from Figure 4.

\section{Summary}

In this paper we compare between the maximum throughputs of IEEE 802.11ax and IEEE 802.11ac in a single user operation mode and in UDP like traffic. In our SU mode the AP transmits continuously to a station using two-level aggregation. Concerning IEEE 802.11ax two flavors are considered, using acknowledgment windows of 256 and 64 MPDUs respectively.

IEEE 802.11 ax outperforms IEEE 802.11 ac by $29 \%$ and $48 \%$ in reliable and unreliable channels respectively. The larger improvement in an unreliable channel is due to the larger number of MPDUs that IEEE 802.11ax enables. Also, a detailed analysis comparing between the two flavors of IEEE 802.11ax is given.

This paper is one of the first to evaluate the performance of IEEE 802.11ax. Other possible scenarios to consider are SU with TCP like traffic where the receiver generates Ack MSDUs such as TCP Ack segments, and MU-MIMO transmissions over the DL and the UL.

\section{References}

[1] IEEE Std. 802.11TM-2016, IEEE Standard for Information Technology-Telecommunications and Information Exchange between Systems-Local and Metropolitan Area Networks-Specific Requirements. Part 11: Wireless LAN Medium Access Control (MAC) and Physical Layer (PHY) Specifications, IEEE, New York, December 2016.

[2] IEEE P802.11axTM/D1.2, Draft Standard for Information Technology-Telecommunications and Information Exchange between Systems-Local and Metropolitan Area Networks-Specific Requirements. Part 11: Wireless LAN Medium Access Control (MAC) and Physical Layer (PHY) Specifications, IEEE, New York, 2016. 
[3] IEEE Std. 802.11acTM-2013, IEEE Standard for Information Technology-Telecommunications and Information Exchange between Systems-Local and Metropolitan Area Networks-Specific Requirements. Part 11: Wireless LAN Medium Access Control (MAC) and Physical Layer (PHY) Specific Requirements. Amendment 4: Enhancements for Very High Throughput for Operation in Bands below 6 GHz, IEEE, New York, 2013.

[4] Perahia, E. and Stacey, R. (2013) Next Generation Wireless LANs: 802.11n and 802.11ac. 2nd Edition, Cambridge Press, Cambridge.

[5] Khorov, E., Kiryanov, A. and Lyakhov, A. (2015) IEEE 802.11ax: How to Build High Efficiency WLANs. 2015 International Conference on Engineering and Telecommunication (EnT), Moscow, 18-19 November 2015, 14-19.

[6] Afaqui, M.S., Villegas, E.G. and Aguilera, E.L. (2016) IEEE 802.11ax: Challenges and Requirements for Future High Efficiency WiFi. IEEE Wireless Communications, PP, 2-9.

[7] Deng, D.J., Chen, K.C. and Cheng, R.S. (2014) IEEE 802.11ax: Next Generation Wireless Local Area Networks. 201410 th International Conference on Heterogeneous Networking for Quality, Reliability, Security and Robustness (QShine), Rhodes, 18-20 August 2014, 77-82. https://doi.org/10.1109/qshine.2014.6928663

[8] Bellalta, B. (2016) IEEE 802.11ax: High-Efficiency WLANs. IEEE Wireless Communications, 23, 38-46. https://doi.org/10.1109/MWC.2016.7422404

[9] Khorov, E., Loginov, V. and Lyakhov, A. (2016) Several EDCA Parameter Sets for Improving Channel Access in IEEE 802.11ax Networks. 2016 International Symposium on Wireless Communication Systems (ISWCS), Poznan, 20-23 September 2016, 419-423.

[10] Lin, W., Li, B., Yang, M., Qn, Q., Yan, Z., Zuo, X. and Yang, B. (2016) Integrated Link-System Level Simulation Platform for the Next Generation WLAN, IEEE 802.11ax. 2016 IEEE Global Communications Conference (GLOBECOM), 1-7.

[11] Karmakar, R., Chattopadhyay, S. and Chakraborty, S. (2017) Impact of IEEE 802.11n/ac PHY/MAC High Throughput Enhancements over Transport/Application Layer Protocols-A Survey. IEEE Communication Surveys and Tutorials.

[12] Sharon, O. and Alpert, Y. (2014) MAC Level Throughput Comparison: 802.11ac vs. 802.11n. Physical Communication, 12, 33-49.

[13] Lemmon, J. (2002) Wireless Link Statistical Bit Error Rate Model. Technical Report 02-934, U.S. Department of Commerce, June 2002. 


\section{Appendix}

In this appendix we show how to derive $X_{o p t}$ in Equation (7) from the term for the throughput in Equation (6).

After some arrangement of Equation (6) we receive the following equation:

$$
F(x)=\frac{L_{D A T A}}{L e n} \cdot \frac{8}{T+O_{P}-P_{D L}} \cdot\left(R_{D L}\left(T-O_{P}\right)-X \cdot O_{M}\right) \cdot(1-B E R)^{\frac{8 \cdot R_{D L} \cdot\left(T-O_{P}\right)}{X}}
$$

The first two terms of Equation (8) are constants and so we need to find the $X$ that maximizes Equation (9):

$$
G(x)=\left(R_{D L}\left(T-O_{P}\right)-X \cdot O_{M}\right) \cdot(1-B E R)^{\frac{8 \cdot R_{D L} \cdot\left(T-O_{P}\right)}{X}}
$$

In order to find this $X$ we derive Equation (9) and compare the derivative to 0 . We receive:

$$
\begin{aligned}
& O_{M} \cdot X^{2}-\left[8 \cdot O_{M} \cdot R_{D L} \cdot\left(T-O_{P}\right) \cdot \ln (1-B E R)\right] X \\
& +8 \cdot R_{D L}^{2} \cdot\left(T-O_{P}\right)^{2} \cdot \ln (1-B E R)=0
\end{aligned}
$$

Solving the quadratic equation, Equation (10), we get that the optimal $X$ is given in Equation (11):

$$
X=\frac{8 \cdot R_{D L} \cdot\left(T-O_{P}\right) \cdot \ln (1-B E R)\left(1-\sqrt{1-\frac{4}{8 \cdot O_{M} \cdot \ln (1-B E R)}}\right)}{2}
$$

We choose the "-" alternative before the square root because we look for a positive $X$ and the term $8 \cdot R_{D L} \cdot\left(T-O_{P}\right) \cdot \ln (1-B E R)$ is negative. We now multiply the $X$ from Equation (11) by the first two constant terms of Equation (8) to receive $X_{\text {opt }}$ in Equation (7).

\section{Submit or recommend next manuscript to SCIRP and we will provide best} service for you:

Accepting pre-submission inquiries through Email, Facebook, LinkedIn, Twitter, etc. A wide selection of journals (inclusive of 9 subjects, more than 200 journals)

Providing 24-hour high-quality service User-friendly online submission system Fair and swift peer-review system Efficient typesetting and proofreading procedure

Display of the result of downloads and visits, as well as the number of cited articles Maximum dissemination of your research work

Submit your manuscript at: http://papersubmission.scirp.org/

Or contact wsn@scirp.org 